\title{
Gerd Strohmeier
}

\section{Minderheitsregierungen in Deutschland auf Bundesebene - Krise oder Chance?}

\section{Ergebnisse eines internationalen Vergleichs}

"Die Sache ist kompliziert«, eröffnete Nikolaus Brender die Berliner Runde im ZDFHauptstadtstudio in Berlin am Abend der Bundestagswahl 2005. Kompliziert war »die Sache« insbesondere deshalb, weil »Schwarz-Gelb « gegen »Rot-Grün« angetreten war, aber weder eine Koalition aus CDU/CSU und FDP noch eine Koalition aus SPD und B'90/Die Grünen eine (absolute) Mehrheit im Bundestag erringen konnte. Der politische Wettbewerb hat zwei konkurrierende Lager, jedoch keinen Sieger hervorgebracht. Fest stand am Wahlabend nur, dass eine andere Koalition gebildet werden musste als im Wahlkampf angekündigt wurde, eine »Kleine Koalition « aus zwei Parteien nicht mehr zustande kommen könnte und die Bundesrepublik sich in gewisser Weise am Rande der Regierbarkeit bewegte. Vor diesem Hintergrund entstand ein regelrechter »Zwang zu einer Großen Koalition «, ${ }^{1}$ dem die großen Parteien widerwillig, aber verantwortungsbewusst nachgaben. Auch bei künftigen Bundestagswahlen könnte - aufgrund der Erosion der Volksparteien, der Dekonzentration des Parteiensystems bzw. der Verfestigung des Fünf-Parteiensystems ${ }^{2}$ - »die Sache kompliziert werden «.

In einem Fünf-Parteiensystem mit einer fehlenden Mehrheit für eine Kleine Koalition aus zwei Parteien gibt es letztlich nur drei Alternativen für die Regierungsbildung: eine »Dreierkoalition «, eine Große Koalition oder eine Minderheitsregierung. Dabei fällt auf, dass die zuletzt genannte Alternative in der politischen, gesellschaftlichen und auch wissenschaftlichen Diskussion kaum als wirkliche Alternative gehandelt wird. So wird die Möglichkeit einer Minderheitsregierung in Politik, Gesellschaft und auch Wissenschaft häufig ignoriert, zum Teil sogar regelrecht tabuisiert oder - wenn sie auf die Agenda gebracht wird - vehement kritisiert. ${ }^{3}$ Die Ressentiments gegenüber einer Minderheits-

1 Helmut Schmidt, Große Koalition - Kleiner Nenner. Vortrag anlässlich der Herbsttagung des Politischen Clubs der Evangelischen Akademie Tutzing, 11.11.2006.

2 Dabei werden CDU und CSU aufgrund der langjährigen Fraktionsgemeinschaft im Bund und der fehlenden Konkurrenz zwischen beiden Parteien - auf analytischer Ebene - als Einheit gewertet.

3 Dies belegt u.a. die zum Teil heftige Auseinandersetzung zum Thema Minderheitsregierung in der Zeitschrift für Parlamentsfragen: Wolfgang Renzsch / Stefan Schieren, »Große Koalition oder Minderheitsregierung: Sachsen-Anhalt als Zukunftsmodell des parlamentarischen Regierungssystems in den neuen Bundesländern? « in: ZParl 28, Nr. 3 (1997), S. 391-407; Winfried Steffani, »Zukunftsmodell Sachsen-Anhalt? Grundsätzliche Bedenken« in: ZParl 28, Nr. 4 (1997), S. 717-722; Wolfgang Renzsch / Stefan Schieren, »Große Koalition ohne Alternative?« 
regierung sind in Deutschland äußerst stark - obwohl beispielsweise in Sachsen-Anhalt zwei Minderheitsregierungen insgesamt acht Jahre verhältnismäßig (!) problemlos regierten. ${ }^{4}$ Auf Bundesebene gilt die Minderheitsregierung jedoch in weiten Teilen von Politik, Gesellschaft und auch Wissenschaft als »Ausnahme [...], die nur in Krisensituationen kurzfristig akzeptabel ist ${ }^{5}{ }^{5}$ bzw. als »unerwünschtes Krisensymptom ${ }^{6}$ oder gar als »Schreckensszenario «. ${ }^{7}$ Die zum Teil nicht vorurteilsfreie Skepsis gegenüber Minderheitsregierungen speist sich im Wesentlichen aus negativen Erfahrungen mit Minderheitsregierungen in der Weimarer Republik und mangelnden Erfahrungen mit Minderheitsregierungen in der Bonner/Berliner Republik. Doch gerade vor diesem Hintergrund erscheint es notwendig, sich intensiv mit den Restriktionen und Implikationen einer Minderheitsregierung in Deutschland auf Bundesebene auseinanderzusetzen. Dabei ist ein Vergleich mit Ländern, in denen häufig Minderheitsregierungen gebildet werden, sinnvoll. Während Minderheitsregierungen in Deutschland einen Ausnahmefall darstellen, bilden sie im internationalen Vergleich einen Normalfall, in skandinavischen Ländern sogar den Regelfall. So werden in Dänemark, Norwegen und Schweden regelmäßig Minderheitsregierungen gebildet und als gängige Form parlamentarischer Regierungsweise akzeptiert. ${ }^{8}$ In Dänemark wurden nach 1945 mit nur wenigen Ausnahmen (Mai 1957 bis November 1960, Februar 1968 bis Oktober 1971 und Januar 1993 bis September 1994) stets Minderheitsregierungen gebildet. ${ }^{9}$ Auch in Schweden wurden zwischen 1945 und 2006 mit nur wenigen Ausnahmen (Oktober 1951 bis Oktober 1957, Januar 1969 bis Januar 1971 und Oktober 1979 bis Mai 1981) stets Minderheitsregierungen gebildet. ${ }^{10}$ In Norwegen formierten sich nach 1945 bis September 1961 Mehrheitsregierungen. Danach wurden bis 2005 mit ebenfalls nur wenigen Ausnahmen (Oktober 1965 bis März 1971 und Juni 1983 bis September 1985) stets Minderheitsregierungen

in: ZParl 29, Nr. 1 (1998), S. 187-188; Winfried Steffani, »Wer trägt die Verantwortung? Wider die wissenschaftliche und politische Verharmlosung des >Magdeburger Modells« in: ZParl 29, Nr. 1 (1998), S. 189-190.

4 Sven Thomas, "Zur Handlungsfähigkeit von Minderheitsregierungen am Beispiel des >Magdeburger Modells« in: ZParl 34, Nr. 4 (2003), S. 792-806, hier: S. 793.

5 Detlef Jahn, »Koalitionen in Dänemark und Norwegen: Minderheitsregierungen als Normalfall « in: Sabine Kropp / Suzanne S. Schüttemeyer / Roland Sturm (Hg.), Koalitionen in Westund Osteuropa, Opladen 2002, S. 219-247, hier: S. 219.

6 Klaus v. Beyme, Die parlamentarischen Regierungssysteme in Europa, München 1970, S. 570.

7 Hans Joachim Veen, "Stabilisierung auf dünnem Eis. Entwicklungstendenzen des Parteiensystems nach der zweiten gesamtdeutschen Wahl in: Heinrich Oberreuter (Hg.), Parteiensystem am Wendepunkt? Wablen in der Fernsebdemokratie, München 1996, S. 182-203, hier: S. 201.

8 Peter Nannestad, »Das politische System Dänemarks« in: Wolfgang Ismayr (Hg.), Die politischen Systeme Westeuropas, Wiesbaden 2009, S. 65-106, hier: S. 77f.

9 Jahn, »Koalitionen in Dänemark und Norwegen: Minderheitsregierungen als Normalfall«, aaO. (FN 5), S. $225 f$.

10 Detlef Jahn, »Das politische System Schwedens in: Ismayr (Hg.), Die politischen Systeme Westeuropas, aaO. (FN 8), S. 107-149, hier: S. 114; http://www.sweden.gov.se/sb/d/4136/a/ 19170, 10.2.2009, zum Teil eigene Berechnungen. 
gebildet. ${ }^{11}$ Allerdings formierten sich in jüngster Zeit in Schweden ${ }^{12}$ und Norwegen ${ }^{13}$ wieder Mehrheitsregierungen. ${ }^{14}$ Dennoch wird deutlich, dass Minderheitsregierungen in Dänemark, Norwegen und Schweden im Gegensatz zu Deutschland »eine selbstverständliche Form des Regierens darstellen $\ll \cdot{ }^{15}$ Vor diesem Hintergrund stellt sich die Frage, ob die - strukturellen und kulturellen - Rahmenbedingungen für die Bildung, Stabilität und Handlungsfähigkeit von Minderheitsregierungen in Deutschland ungünstiger sind als in Dänemark, Norwegen und Schweden.

Im Folgenden wird zunächst die aktuelle Situation in Deutschland dargestellt, unter der nur eine Große Koalition, Dreierkoalition oder Minderheitsregierung als Alternative für die Regierungsbildung in Frage kommt: ein Fünf-Parteiensystem mit einer fehlenden Mehrheit für eine Kleine Koalition aus zwei Parteien. Dabei wird deutlich gemacht, dass eine Dreierkoalition eine unwahrscheinliche bzw. unbeständige sowie eine Große Koalition eine nicht unumstrittene bzw. unproblematische Konstellation darstellt - und daher die grundsätzliche Diskussion über die Minderheitsregierung als Regierungsalternative sinnvoll ist. Anschließend wird die Kreation bzw. Einsetzbarkeit, Stabilität bzw. Abberufbarkeit und Funktionalität bzw. Handlungsfähigkeit einer Minderheitsregierung in Deutschland auf Bundesebene untersucht. Dabei wird jeweils geklärt, ob die strukturellen und kulturellen - Rahmenbedingungen für Minderheitsregierungen in Deutschland (erwartungsgemäß) ungünstiger sind als in Dänemark, Norwegen und Schweden.

11 Hermann Groß / Walter Rothholz, »Das politische System Norwegens « in: Ismayr (Hg.), Die politischen Systeme Westeuropas, aaO. (FN 8), S. 151-193, hier: S. 165; Jahn, »Koalitionen in Dänemark und Norwegen: Minderheitsregierungen als Normalfall«, aaO. (FN 5), S. $223 f$.

12 Dort wurde - in Folge der abnehmenden Dominanz der Sozialdemokratischen Arbeiterpartei und der zunehmenden Einigkeit der bürgerlichen Parteien - nach der Reichstagswahl 2006 die von der Linkspartei und der Umweltpartei unterstützte Minderheitsregierung der Sozialdemokratischen Arbeiterpartei durch eine bürgerliche Mehrheitsregierung, bestehend aus Moderater Sammlungspartei, Liberaler Volkspartei, Zentrumspartei und Christdemokratischer Partei, abgelöst (Jahn, »Das politische System Schwedens«, aaO. (FN 10), S. 117ff.).

13 Dort wurde - nachdem zunehmend relativ schwache Minderheitsregierungen gebildet wurden und die Arbeiterpartei ihr »Muster der Koalitionsvermeidung « aufgab - nach der Parlamentswahl 2005 die von der Fortschrittspartei unterstützte Minderheitsregierung der bürgerlichen Koalition, bestehend aus Konservativer Partei, Christlicher Volkspartei und Liberaler Partei, durch eine Mehrheitsregierung, bestehend aus Arbeiterpartei, Sozialistischer Linkspartei und Zentrumspartei, abgelöst (Jahn, »Koalitionen in Dänemark und Norwegen: Minderheitsregierungen als Normalfall«, aaO. (FN 5), S. 227ff.).

14 Dabei wird deutlich, dass die Option für eine Mehrheitsregierung - sofern sie sich bietet - in den für Minderheitsregierungen bekannten skandinavischen Ländern durchaus genutzt wird (dort also auch die Vorzüge einer Mehrheitsregierung geschätzt werden).

15 Wolfgang Ismayr, »Die politischen Systeme Westeuropas im Vergleich « in: ders. (Hg.), Die politischen Systeme Westeuropas, aaO. (FN 8), S. 9-63, hier: 32. 


\section{Die Minderheitsregierung als Alternative (zur Dreierkoalition und Großen Koalition)}

Grundsätzlich ist davon auszugehen, dass Parteien in parlamentarischen Regierungssystemen $^{16}$ - v.a. zur Sicherung der Regierungsstabilität und -funktionalität - bestrebt sind, Mehrheitsregierungen zu bilden. Auf dieser Annahme basieren auch die klassischen Koalitionstheorien. ${ }^{17}$ Sie sagen die Bildung von Mehrheitskoalitionen für den Fall voraus, dass keine Partei eine absolute Mehrheit der Parlamentsmandate erhält, was in parlamentarischen Regierungssystemen mit Verhältniswahl die Regel ist. Die Regierungsbzw. Koalitionsbildung kann sich jedoch als schwierig erweisen, wenn nicht alle arithmetischen Koalitionsmöglichkeiten zugleich politische Koalitionsmöglichkeiten darstellen. So scheiden arithmetische Koalitionsmöglichkeiten als politische Koalitionsmöglichkeiten aus, wenn sie Parteien umfassen, die von den anderen Parteien dieser Koalitionsvariante grundsätzlich als nicht koalitionsfähig bzw. »koalitionsunwürdig « eingestuft werden; eine zu kleine inhaltliche Schnittmenge bzw. eine zu große programmatische Spannbreite umfassen; aus einer zu großen Anzahl von (unterschiedlichen) Parteien bestehen; eine zu kleine bzw. instabile Mehrheit im Parlament besitzen etc. Dabei wird deutlich, dass Parteiensysteme bzw. deren spezifische Strukturen einen äußerst großen Einfluss auf die Regierungsbildung haben.

In skandinavischen Ländern haben die - verhältnismäßig ähnlichen - Strukturen der dortigen Parteiensysteme maßgeblich zur Bildung von Minderheitsregierungen beigetragen. $\mathrm{Zu}$ diesen Strukturen zählen eine (in den einzelnen Ländern zum Teil mehr, zum Teil weniger stark ausgeprägte) Dekonzentration, ${ }^{18}$ Blockbildung, ${ }^{19}$ Fragmentierung und Uneinigkeit des »bürgerlichen Blocks $\aleph^{20}$ (und zum Teil damit einher gehende Do-

16 Als parlamentarische Regierungssysteme gelten im Rahmen dieses Beitrags - in Anlehnung an Winfried Steffani - alle Regierungssysteme, in denen das Parlament die Regierung grundsätzlich aus politischen Gründen abberufen kann (Winfried Steffani, »Parlamentarisch-präsidentielle „Mischsysteme“? Bemerkungen zum Stand der Forschung in der Politikwissenschaft « in: Otto Luchterhandt (Hg.), Neue Regierungssysteme in Osteuropa und der GUS, Probleme der Ausbildung stabiler Machtinstitutionen, Berlin 2002, S. 17-66, hier: S. 49ff.).

17 Sabine Kropp / Suzanne S. Schüttemeyer / Roland Sturm, »Koalitionen in West- und Osteuropa « in: dies. (Hg.), Koalitionen in West- und Osteuropa, aaO. (FN 5), S. 7-41.

18 Zur Beschreibung (der Dekonzentration) des Parteiensystems wird im Folgenden nur die Anzahl der (Parlaments-)Parteien - und nicht etwa die effektive Anzahl der (Parlaments-)Parteien (Markku Laasko / Rein Taagepera, »Effective Number of Parties. A Measure with Application to West Europe« in: Comparative Political Studies 12, Nr. 1 (1979), S. 3-27, hier: S. 3ff.) - wiedergegeben, obwohl diese allein noch nichts über die konkreten parlamentarischen Machtverhältnisse und Regierungsoptionen aussagt - was aber z.B. die effektive Anzahl der (Parlaments-)Parteien letztlich auch nicht tut (vgl. auch Jahn, »Koalitionen in Dänemark und Norwegen: Minderheitsregierungen als Normalfall«, aaO. (FN 5), S. 246).

19 Eine besonders starke Blockbildung ist generell in Norwegen und in Schweden zu beobachten. Dabei ist hervorzuheben, dass die Blockgrenzen in Norwegen und Schweden - im Gegensatz zu Dänemark - in der Regel (in Norwegen zumindest bis 2005) "Demarkationslinien für die Koalitionspolitik « (ebd., S. 222ff.) darstellten - allerdings nicht für die »Unterstützungspolitik « bei Minderheitsregierungen.

20 Eine besonders starke Uneinigkeit der bürgerlichen Parteien ist in Norwegen und (jedenfalls bis 2006) in Schweden zu beobachten. 
minanz der sozialdemokratischen Partei $)^{21}$ sowie Koalitionsunwürdigkeit ${ }^{22}$ bestimmter Parteien.

In Dänemark existierte zwischen 1945 und 1971 ein Sechs- bis Sieben-Parteiensystem (in dem das »traditionelle Vierparteiensystem «, das sich etwa um 1920 konsolidierte, dominierte). Nachdem sich die Anzahl der im Parlament vertretenen Parteien 1971 kurzfristig auf fünf reduzierte, stieg sie 1973 - vor allem aufgrund der umstrittenen EG-Mitgliedschaftsfrage - auf zehn Parteien an (wobei das »traditionelle Vierparteiensystem« stark erodierte). Das danach stark im Fluss befindliche bzw. größeren Veränderungen $^{23}$ unterworfene Parteiensystem bildet seit der Parlamentswahl von 2007 ein AchtParteiensystem. Dazu zählen die (als koalitionsunwürdig eingestufte) Einheitsliste, die Sozialistische Volkspartei sowie die Sozialdemokratische Partei im »linken Block «, die Radikal-liberale Partei sowie die Neue Allianz in gewisser Weise im »Mittel-Block « und die Konservative Partei, die Liberale Partei sowie die (als koalitionsunwürdig eingestufte) Dänische Volkspartei im »rechten Block «. ${ }^{24}$

In Norwegen existierte zwischen 1945 bis 1973 ein Fünf- bis Sechs-Parteiensystem (dessen Keim im Wesentlichen um 1920 zu finden ist). Dieses differenzierte sich danach - auch vor allem aufgrund der umstrittenen EG-Mitgliedschaftsfrage - weiter aus und bildet heute nach einigen Schwankungen ein Sieben-Parteiensystem. Dazu zählen die (lange Zeit als koalitionsunwürdig eingestufte) Sozialistische Linkspartei sowie die Arbeiterpartei im »linken Block « und die Christliche Volkspartei, die Zentrumspartei, die Konservative Partei, die Liberale Partei sowie die (als koalitionsunwürdig eingestufte) Fortschrittspartei im (stark fragmentierten) $»$ rechten Block « ${ }^{25}$

In Schweden existierte von den 1920er-Jahren an lange Zeit ein Fünf-Parteiensystem, das sich Ende der 1980er-Jahre auszudifferenzieren begann und in den 1990er-Jahren auf ein Sieben-Parteiensystem anwuchs. Dazu zählen die (als koalitionsunwürdig eingestufte) Linkspartei, die Sozialdemokratische Arbeiterpartei sowie die Umweltpartei im »linken Block« und die Liberale Volkspartei, die Zentrumspartei, die Christdemokratische

21 Die Dominanz der sozialdemokratischen Partei ist allerdings in Norwegen und Schweden einem Erosionsprozess ausgesetzt und in Dänemark mittlerweile sogar gänzlich verschwunden: Während die sozialdemokratische Partei in Norwegen und Schweden immer noch die meisten Wählerstimmen auf sich vereinen kann, ist diese in Dänemark 2001 von der Liberalen Partei als stimmenstärkste Partei abgelöst worden.

22 Die Koalitionsunwürdigkeit impliziert jedoch grundsätzlich keine »Unterstützungsunwürdigkeit « - schließt also die Unterstützung einer Minderheitsregierung durch als koalitionsunwürdig eingestufte Parteien generell nicht aus.

23 Dazu zählt im letzten Jahrzehnt neben dem Bedeutungsverlust der Sozialdemokratischen Partei v.a. das Verschwinden der Zentrumsdemokraten und der Christlichen Volkspartei als Parteien der Mitte, die Etablierung der Dänischen Volkspartei und das Aufkommen der Neuen Allianz (Nannestad, »Das politische System Dänemarks«, aaO. (FN 8), S. 94).

24 Ebd., S. 88ff.; Jahn, »Koalitionen in Dänemark und Norwegen: Minderheitsregierungen als Normalfall«, aaO. (FN 5), S. 225f.

25 Groß / Rothholz, »Das politische System Norwegens «, aaO. (FN 11), S. 173 ff.; Jahn, »Koalitionen in Dänemark und Norwegen: Minderheitsregierungen als Normalfall«, aaO. (FN 5), S. $222 \mathrm{ff}$. 
Partei sowie die Moderate Sammlungspartei im (inhaltlich divergierenden) »rechten Block ${ }^{26}$

In Deutschland hat sich das Parteiensystem in jüngster Zeit in einigen Wesensmerkmalen den Strukturen skandinavischer Parteiensysteme angenähert. So existiert seit der Bundestagswahl 2005 auf Bundesebene ein dekonzentriertes Parteiensystem mit zwei »Blöcken« sowie einer als koalitionsunwürdig eingestuften Partei (Die Linke) - die eine rot-grüne oder schwarz-gelbe Mehrheit verhindert. In der Folge reduzierten sich nach der Bundestagswahl 2005 sämtliche arithmetischen Koalitionsmöglichkeiten auf eine politische Koalitionsmöglichkeit: die Große Koalition. Die Bildung einer »Ampel-Koalition «, bestehend aus SPD, B'90/Die Grünen und FDP, war letztlich ebenso unrealistisch wie die Bildung einer »Jamaika-Koalition «, bestehend aus CDU/CSU, FDP und B'90/Die Grünen. Auch in Zukunft ist eine Dreierkoalition eine unwahrscheinliche bzw. - sollte sie wider erwarten doch gebildet werden - unbeständige Konstellation. Auf der Grundlage der Koalitionsforschung lassen sich sowohl »policy-seeking « Motive als auch »vote-seeking « Motive der Parteien gegen die Bildung bzw. gegen die Funktionalität einer Dreierkoalition vorbringen. Zum einen würde dem Streben der Parteien nach einer möglichst ungefilterten Durchsetzung programmatischer Forderungen in Dreierkoalitionen deutliche Grenzen gesetzt werden, da die »policy distance « zwischen den Parteien bzw. die »range « innerhalb einer Dreierkoalition äußerst groß wäre. Zum anderen würde - nicht zuletzt dadurch - das Streben der Parteien nach einer Maximierung von Wählerstimmen erheblich erschwert werden, da es für die Parteien in einer Dreierkoalition äußerst schwierig wäre, Profil zu gewinnen bzw. nicht an Profil zu verlieren. Dies gilt v.a. für die kleinen Parteien. So könnten in einer Ampel-Koalition entweder die Liberalen als »Mehrheitsbeschaffer« von Rot-Grün oder B'90/Die Grünen als »Annex « der SPD wahrgenommen werden. Das größere Risiko würde in einer Ampel-Koalition sicherlich die FDP tragen, da diese am stärksten von den bisherigen Mustern der Parteienkonkurrenz abweichen müsste. Das Gleiche gilt natürlich für B'90/Die Grünen in einer JamaikaKoalition. Vor diesem Hintergrund überrascht es nicht, dass nach der Bundestagswahl 2005 nur eine Große Koalition als Alternative zur Regierungsbildung in Frage kam, wenngleich es sich hierbei grundsätzlich um eine in vielerlei Hinsicht problematische Konstellation handelt. Dies klang bereits in der Regierungserklärung der ersten Großen Koalition an: »Die stärkste Absicherung gegen einen möglichen Missbrauch der Macht ist der feste Wille der Partner der Großen Koalition, diese nur auf Zeit, also bis zum Ende dieser Legislaturperiode[,] fortzuführen. «27 Mit diesen Worten beschwor Kurt Georg

26 Die rechtspopulistische »Neue Demokratie «, die 1991 in den Reichstag einzog und als koalitionsunwürdig eingestufte Partei die Bildung einer Mehrheitsregierung verhinderte, konnte sich bei nachfolgenden Wahlen nicht mehr durchsetzen (Jahn, »Das politische System Schwedens«, aaO. (FN 10), S. 126; Heinrich Pehle, »Koalitionen in Finnland und Schweden: Fortbestand der Unterschiede trotz Angleichung der Systeme« in: Kropp / Schüttemeyer / Sturm (Hg.), Koalitionen in West- und Osteuropa, aaO. (FN 5), S. 197-217, hier: S. 199ff.).

27 Kurt Georg Kiesinger, »Regierungserklärung des Bundeskanzlers am 13.12.1966 vor dem Deutschen Bundestag in Bonn « in: Hans Ulrich Behn (Hg.), Die Regierungserklärungen der Bundesrepublik Deutschland, München 1971, S. 185-204, hier: S. 186. 
Kiesinger das Ende des von ihm angeführten Regierungsbündnisses, ehe es richtig begonnen hatte, nicht zuletzt, um auf die Kritik zu reagieren, die einem derartigen Bündnis entgegengebracht wurde - und zum Teil immer noch wird. Von einigen sachkundigen Beobachtern wird eine Große Koalition sogar als »Widerspruch zum parlamentarischen System «, ${ }^{28} »$ unheilige Allianz «, ${ }^{29}$ »Art von demokratischem Sündenfall ${ }^{30}$ oder gar »Gift für die Demokratie «11 etikettiert.

Der Kern des demokratietheoretischen Problems einer Großen Koalition besteht darin, dass die beiden »Hauptkonkurrenten « im politischen Wettbewerb ein Regierungsbündnis eingehen. Dabei handelt es sich häufig - wie nach der Bundestagswahl 2005 um ein Regierungsbündnis, das weder von den Parteien noch von den Wählern (mehrheitlich) gewollt war. So haben zwar 69,4 Prozent der Wähler die Parteien der Großen Koalition gewählt, aber nicht einmal halb so viele Wähler (33 Prozent) eine Große Koalition gewollt. Noch problematischer ist indes, dass eine Große Koalition de facto nicht abgewählt werden kann: Setzen die Regierungsparteien die Große Koalition fort, ist de facto überhaupt kein Regierungswechsel möglich; tun sie dies nicht, ist de facto nur ein unvollständiger Regierungswechsel, d.h. ein Regierungswechsel in Form eines Partnerwechsels, möglich. Die Opposition zu einer Großen Koalition ist also nicht oder - bestenfalls - nur zum Teil das, was eine demokratische Opposition sein sollte: eine »Regierung im Wartestand «. ${ }^{32}$ Durch die Verhinderung bzw. Einschränkung des Wechselspiels zwischen Regierung und Opposition wird in gewisser Weise der Prozess der demokratischen Wahl entwertet bzw. - dramatischer ausgedrückt - das »Lebenselixier demokratischer Regierungsweise «33 vernichtet. Schließlich »lebt « die repräsentative Demokratie »vom Wettbewerb der Ideen und Konzepte, von der Überzeugung der Wähler, wirklich eine Wahl zu haben und mit ihrer Stimme über die Zukunft mit zu entscheiden « ${ }^{34}$ Daran schließt das Problem an, dass unter Umständen gerade die Unzufriedenheit mit der Großen Koalition deren Fortbestand sichert: Je mehr - unter den gegenwärtigen Bedingung des Parteiensystems - aus den kleinen Parteien, speziell natürlich der als koalitionsunwürdig eingestuften Linkspartei, ein »Sammelbecken für die mit der Regierung unzu-

28 Renzsch / Schieren, »Große Koalition oder Minderheitsregierung: Sachsen-Anhalt als Zukunftsmodell des parlamentarischen Regierungssystems in den neuen Bundesländern? «, aaO. (FN 3), S. 403.

29 Harold Rasch, »Die Unheilige Allianz. Ein Jahr >Große Koalition«« in: Blätter für deutsche und internationale Politik 12, Nr. 11 (1967), S. 1115-1126.

30 Peter Graf Kielmannsegg, »Mehrheiten sind nicht mehr garantiert « in: Frankfurter Allgemeine Zeitung, 23.8.2002, S. 9.

31 Renate Köcher, »Gift für die Demokratie, Allensbach-Analyse« in: FAZ.NET, http:// www.faz.net, 19.10.2006.

32 Renzsch / Schieren, »Große Koalition oder Minderheitsregierung: Sachsen-Anhalt als Zukunftsmodell des parlamentarischen Regierungssystems in den neuen Bundesländern?«, aaO. (FN 3), S. 392.

33 Werner Kaltefleiter, »Die Große Koalition. Verfassungspolitische Aufgaben und Probleme« in: APuZ, (1967) 18-19, S. 3-23.

34 Köcher, »Gift für die Demokratie, Allensbach-Analyse«, aaO. (FN 31). 
friedene Wählerschaft « ${ }^{35}$ wird, desto geringer wird die Wahrscheinlichkeit, dass die Große Koalition von einer (stabilen) Kleinen Koalition abgelöst werden kann. Dies zeigt auch die jüngste Entwicklung in Österreich, wo im Prinzip ganz ähnliche Verhältnisse wie in Deutschland vorzufinden sind. Dort führte das schlechte Funktionieren der 2007 gebildeten Großen Koalition 2008 zu einer vorzeitigen Neuwahl, diese zu einer »Abstrafung « der großen Parteien und diese letztlich zur Notwendigkeit einer Neuauflage der Großen Koalition. In Deutschland zeigt die Entwicklung der Parteipräferenzen seit der Bundestagswahl 2005 jedenfalls eine zunehmende Erosion der Volksparteien bzw. Dekonzentration des Parteiensystems, welche in Zukunft erneut die Bildung einer Großen Koalition »erzwingen « könnte - die durch die Opposition nicht (vollständig) abgelöst werden kann. Eine Große Koalition verhindert allerdings nicht nur eine »regierungsfähige Opposition «, also eine Opposition, die eine faktische Regierungsalternative darstellt, sondern auch eine handlungsfähige Opposition, d.h. eine Opposition, die eine effektive bzw. uneingeschränkte Kontrollinstanz darstellt. So kann die parlamentarische Opposition zur zweiten Großen Koalition in Deutschland z.B. kein abstraktes Normenkontrollverfahren einleiten (wofür ein Drittel der Mitglieder des Bundestags erforderlich ist; vgl. Art. 93 Abs. 1 Nr. 2 GG) und nur mit vereinten Kräften, d.h. mit den Stimmen von FDP, B'90/Die Grünen und Linkspartei, einen Untersuchungsausschuss einsetzen (wofür ein Viertel der Mitglieder des Bundestags erforderlich ist; vgl. Art. 44 Abs. 1 Satz 1 GG). Das Fehlen einer effektiven parlamentarischen Opposition kann unter Umständen dazu führen, »dass aus systemloyaler Opposition gegen die Regierung Opposition gegen das System entsteht « ${ }^{36}$ d.h. dass die Kritik an der Großen Koalition - wie in Österreich während der letzten Großen Koalitionen - zu massiven Stimmenzuwächsen von extremen bzw. populistischen Parteien führt oder - wie in Deutschland während der ersten Großen Koalition - in einer starken außerparlamentarischen Opposition mündet. ${ }^{37}$ Eine Große Koalition reduziert allerdings nicht nur die Bedeutung sowie die Einflussmöglichkeiten der parlamentarischen Opposition, sondern auch die von Teilen der Regierungsfraktionen bzw. einzelner Abgeordneter innerhalb der Regierungsfraktionen. Schließlich kann eine Regierung, die für Beschlüsse eine einfache bzw. absolute Mehrheit der Parlamentsmandate benötigt, aber über eine qualifizierte Mehrheit der Parlamentsmandate verfügt, problemlos auf eine große Anzahl von »Abweichlern« in den eigenen Reihen - und deren Meinung - verzichten. So nimmt mit einer Großen Koalition in gewisser Weise die »Exekutivlastigkeit« des Parlaments zu und die Bedeutung des einzelnen Abgeordneten ab. Die Tatsache, dass eine Große Koalition eine über-

35 Kaltefleiter, »Die Große Koalition. Verfassungspolitische Aufgaben und Probleme«, aaO. (FN 33), S. 7.

36 Renzsch / Schieren, »Große Koalition oder Minderheitsregierung: Sachsen-Anhalt als $\mathrm{Zu}-$ kunftsmodell des parlamentarischen Regierungssystems in den neuen Bundesländern?«, aaO. (FN 3), S. 405.

37 Bei drei kleinen sowie äußerst heterogenen Oppositionsparteien - wie zur Zeit der zweiten Großen Koalition in Deutschland - ist allerdings zu erwarten, dass die Unzufriedenheit der Wähler mit der Regierung weitgehend innerhalb des Parteiensystems »aufgefangen « wird und nicht - wie zur Zeit der ersten Großen Koalition in Deutschland, in der nur die FDP als Oppositionspartei fungierte - in den außerparlamentarischen Raum »entweicht «. 
aus große Mehrheit zur Umsetzung von Reformvorhaben hat, bedeutet allerdings nicht zwingend, dass sie "große« Reformvorhaben umsetzt. Eine im Vergleich zu Kleinen Koalitionen erhöhte Reformtätigkeit ist nur im Bereich des Verfassungsrechts wahrscheinlich (und empirisch eindeutig feststellbar), ${ }^{38} \mathrm{da}$ Verfassungsänderungen grundsätzlich der Zustimmung beider großer Parteien bzw. Fraktionen bedürfen und diese im Rahmen einer Regierungskoalition besser zu erreichen ist als bei einer Regierungs-Oppositions-Konstellation. Im Bereich des einfachen Rechts ist eine im Vergleich zu Kleinen Koalitionen erhöhte Reformtätigkeit (zumindest auf lange Sicht) unwahrscheinlich, da sich die Regierungsparteien einer Großen Koalition - im Gegensatz zu den Regierungsparteien einer Kleinen Koalition - in einem nahezu permanenten und prinzipiell unauflösbaren politischen Wettbewerbsverhältnis befinden und sich in der Folge "gegeneinander und gegen die Regierung als Ganzes profilieren « ${ }^{39}$ müssen. ${ }^{40}$

Zusammengefasst betrachtet bildet eine Große Koalition eine nicht unproblematische Konstellation, die vor dem Hintergrund demokratietheoretischer Überlegungen durchaus so bewertet werden kann, wie eine Minderheitsregierung häufig eingeschätzt wird: als »Ausnahme [...], die nur in Krisensituationen kurzfristig akzeptabel ist «. ${ }^{41}$

\section{Kreation bzw. Einsetzbarkeit einer Minderheitsregierung}

Die Bildung einer Minderheitsregierung wird durch das Grundgesetz eindeutig erschwert. So ist die Wahl eines Bundeskanzlers mit der relativen Mehrheit im Bundestag nur als ultima ratio möglich und dessen Ernennung vom Ermessen des Bundespräsidenten abhängig. Nach Art. 63 Abs. 1 GG wird der Bundeskanzler - in der so genannten ersten Stufe des Kanzlerwahlverfahrens - »auf Vorschlag des Bundespräsidenten vom Bundestage ohne Aussprache gewählt«. Dabei ist gewählt und muss anschließend vom Bundespräsidenten ernannt werden, »wer die Stimmen der Mehrheit der Mitglieder des Bundestages [eine absolute Mehrheit] auf sich vereinigt « (Art. 63 Abs. 2 Satz 1 GG). Wird der vom Bundespräsidenten vorgeschlagene Kandidat nicht mit der absoluten Mehrheit gewählt, kann der Bundestag - in der so genannten zweiten Stufe des Kanzlerwahlverfahrens - »binnen vierzehn Tagen nach dem Wahlgange mit mehr als der Hälfte seiner Mitglieder einen Bundeskanzler wählen«(Art. 63 Abs. 3 GG). Gelingt dies innerhalb des festgelegten Zeitraums (in beliebig vielen Wahlgängen) nicht, »findet unverzüglich ein neuer Wahlgang statt, in dem gewählt ist, wer die meisten Stimmen erhält « (Art. 63 Abs. 4 Satz 1 GG). Erst dann - in der so genannten dritten Stufe des Kanzlerwahlverfahrens - ist die Wahl eines Kandidaten mit relativer Mehrheit und dessen Ernennung

38 So fallen die meisten bzw. umfangreichsten Verfassungsänderungen in Deutschland - mit Ausnahme der nach der Wiedervereinigung beschlossenen Verfassungsänderungen (die zwangsläufig vorgenommen werden mussten) - in die Amtszeiten der Großen Koalitionen.

39 Ebd., S. 404.

40 Vgl. auch Gerd Strohmeier, »Große Koalitionen in Deutschland und Österreich« in: ZPol 19, Nr. 1 (2009), S. 5-37.

41 Jahn, »Koalitionen in Dänemark und Norwegen: Minderheitsregierungen als Normalfall«, aaO. (FN 5), S. 219. 
durch den Bundespräsidenten möglich: »Vereinigt der Gewählte die Stimmen der Mehrheit der Mitglieder des Bundestages auf sich, so muss der Bundespräsident ihn binnen sieben Tagen nach der Wahl ernennen. Erreicht der Gewählte ${ }^{42}$ diese Mehrheit nicht, so hat der Bundespräsident binnen sieben Tagen entweder ihn zu ernennen oder den Bundestag aufzulösen «(Art. 63 Abs. 4 Satz 2 und 3 GG). Sollte also bei dem letzten Wahlgang wieder kein Kandidat eine absolute Mehrheit bzw. ein Kandidat nur eine relative Mehrheit erreichen, tritt eine der wenigen Situationen ein, in denen der Bundespräsident einen politischen Gestaltungsspielraum hat bzw. nach seinem politischen Ermessen entscheiden kann..$^{43}$ So ist die Entscheidung des Bundespräsidenten, nach dem letzten Wahlgang entweder einen Minderheitskanzler zu ernennen oder eine Neuwahl herbeizuführen, »vollständig und allein in sein eigenes politisches Ermessen gelegt $«{ }^{44}$ Dabei wird allerdings erwartet, dass der Bundespräsident eine Einschätzung vornimmt, ob die Ernennung des Minderheitskanzlers - und damit die Bildung einer möglicherweise handlungsunfähigen Regierung - oder die Herbeiführung einer Neuwahl - und damit eine Phase der »Unregierbarkeit« bis zur Neuwahl und möglicherweise ebenso unklare Mehrheitsverhältnisse nach der Neuwahl - in der aktuellen Situation das "geringere Übel« bzw. die »kleinere Gefahr« darstellt. Dass eine Minderheitsregierung (formell) nur in der letzten Stufe des Kanzlerwahlverfahrens, also nach diversen Versuchen, einen Mehrheitskanzler zu etablieren, und nur mit Zustimmung des Bundespräsidenten, also nach einer Prüfung der Vorteile einer Minderheitsregierung gegenüber einer Neuwahl, installiert werden kann, macht deutlich, dass der Parlamentarische Rat - unter dem Einfluss der »Weimarer Erfahrungen « - die Minderheitsregierung prinzipiell als (krisenhafte) Ausnahmeerscheinung gesehen hat und daher »fast mit allen verfassungsrechtlichen Mitteln stabile Regierungsmehrheiten sicherstellen wollte ${ }^{45}$

Ein internationaler Vergleich der Regierungsbildung in parlamentarischen Regierungssystemen zeigt, dass das in Deutschland praktizierte Verfahren eine sehr große Hürde für und damit eine entsprechend starke Absicherung gegen die Einsetzung einer Minderheitsregierung impliziert. So ist nur in wenigen Ländern zur Einsetzung des Regierungschefs bzw. der Regierung im Rahmen einer Wahl oder einer (der Ernennung

42 Fraglich ist allerdings, ob es einen Gewählten geben kann, wenn bei nur einem Kandidaten mehr Nein- als Ja-Stimmen abgegeben werden. Es ist davon auszugehen, dass in diesem Fall wie auch bei einer sich verfestigenden Pattsituation in der dritten Stufe des Kanzlerwahlverfahrens oder einem aufgrund fehlender Kandidaten bzw. Vorschläge Ausbleiben der dritten Stufe des Kanzlerwahlverfahrens (Arnd Uhle, »Art. 63 GG« in: Bruno Schmidt-Bleibtreu / Franz Klein, GG Kommentar zum Grundgesetz, 2008, Rdnr. 25ff.) - eine Auflösung des Bundestags seitens des Bundespräsidenten erfolgt.

43 Gerd Strohmeier, »Der Bundespräsident: Was er kann, darf und muss bzw. könnte, dürfte und müsste in: ZfP 55, Nr. 2 (2008), S. 175-198, hier: S. 177.

44 Roman Herzog, »Art. 63 GG « in: Theodor Maunz / Günter Dürig, Grundgesetz Kommentar, Lfg. 21, Rdnr. 40.

45 Ebd., Rdnr. 53.

ZfP 56. Jg. 3/2009 
bzw. Designierung folgenden) Vertrauensabstimmung eine absolute Mehrheit ${ }^{46}$ erforderlich: ${ }^{47}$ in Bulgarien, Makedonien, Slowenien und Spanien (bei einer Wahl) sowie Kroatien, Lettland, Moldova und der Ukraine (bei einer Vertrauensabstimmung). Von entscheidender Bedeutung ist jedoch, was in den jeweiligen Ländern zu geschehen hat, wenn (letztlich) keine absolute Mehrheit zustande kommt: ob dann, wie z.B. in Bulgarien, das Staatsoberhaupt die Pflicht hat, das Parlament aufzulösen, oder, wie z.B. in Deutschland, das Staatsoberhaupt das Recht hat, zwischen einer Parlamentsauflösung und der Ernennung eines Minderheitskanzlers zu entscheiden, oder, wie z.B. in Spanien, eine einfache Mehrheit genügt. Dabei wird deutlich, dass in Spanien die Hürde für die Einsetzung einer Minderheitsregierung niedriger ist als in Deutschland oder Bulgarien. In vielen Ländern ist grundsätzlich nur eine einfache Mehrheit zur Einsetzung der Regierung erforderlich (und damit die Einsetzung einer Minderheitsregierung von Beginn an einfacher als in Deutschland): in Irland, Finnland und Ungarn (bei einer Wahl) sowie in Italien, ${ }^{48}$ Griechenland, Belgien und den meisten osteuropäischen Ländern (bei einer Vertrauensabstimmung). Prinzipiell noch unproblematischer erscheint die Bildung einer Minderheitsregierung unter den Bedingungen des in Westeuropa weit verbreiteten und v.a. in Skandinavien vorherrschenden »negativen Parlamentarismus «, d.h. wenn zur Einsetzung des Regierungschefs bzw. der Regierung keine Mehrheit dafür stimmen muss, sondern nur keine Mehrheit dagegen stimmen darf. Allerdings ist es - rein arithmetisch betrachtet - egal, ob eine einfache (!) Mehrheit für die Regierung stimmen muss oder nicht gegen die Regierung stimmen darf. Dennoch lassen sich grundsätzlich gewisse Unterschiede ausmachen. So erfolgt im Rahmen des »negativen Parlamentarismus « meist keine obligatorische Abstimmung bei der Einsetzung der Regierung - und macht es für »Nicht-Regierungsparteien « unter Umständen durchaus einen Unterschied, im Rahmen einer obligatorischen Wahl bzw. Vertrauensabstimmung oder im Rahmen eines möglichen Misstrauensvotums gegen die Regierung zu stimmen. Jedenfalls kann in Dänemark und Norwegen der Regierungschef bzw. die Regierung vom Monarchen ohne jedwede parlamentarische Beteiligung eingesetzt und nur in Form eines eingebrachten Misstrauensvotums mit einfacher Mehrheit gestürzt werden. ${ }^{49}$ Etwas anders verhält es sich in Schweden, wo eine obligatorische (Vertrauens-)Abstimmung zu erfolgen hat. Dort ist die arithmetische Hürde für die Etablierung einer Minderheitsregierung allerdings am

46 Allerdings existieren unterschiedliche Bezugsgrößen für absolute Mehrheiten. So ist in Deutschland eine absolute Mehrheit aller Abgeordneten und in Bulgarien eine absolute Mehrheit der anwesenden Abgeordneten (Art. 81 Abs. 2 der bulgarischen Verfassung) erforderlich, die sich natürlich bei vollzähligem Erscheinen (womit im Zusammenhang mit der Einsetzung der Regierung prinzipiell zu rechnen ist) mit der absoluten Mehrheit aller Abgeordneten deckt.

47 Eine zum Teil - v.a. in osteuropäischen Ländern - mögliche und praktizierte Einflussnahme des Staatsoberhaupts auf den (»materiellen«) Regierungsbildungsprozess wird an dieser Stelle ausgeblendet.

48 Dort müssen beide Kammern des Parlaments (in getrennter Abstimmung) ein positives Votum abgeben.

49 Dadurch können Minderheitsregierungen nach Parlamentswahlen ohne jedwede (direkte) parlamentarische Beteiligung »weiteramtieren « bzw. »einfach überleben« (Jahn, »Koalitionen in Dänemark und Norwegen: Minderheitsregierungen als Normalfall«, aaO. (FN 5), S. 244). 
niedrigsten, da zur Ablehnung der Regierung eine absolute Mehrheit des Reichstags gegen den vom Reichstagspräsidenten vorgeschlagenen Ministerpräsidenten stimmen muss. ${ }^{50}$

Der internationale Vergleich macht deutlich, dass die verfassungsrechtlichen Rahmenbedingungen für die Bildung einer Minderheitsregierung in Deutschland verhältnismäßig ungünstig sind, während sie in den skandinavischen Ländern, in denen vermehrt Minderheitsregierungen gebildet werden, sehr günstig sind. Allerdings kann die sehr große Hürde für die Bildung einer Minderheitsregierung in Deutschland umgangen werden, wenn sich ein Teil der Opposition bereit erklärt, einem Minderheitskanzler ins Amt zu verhelfen, d.h. bei der Wahl (!) zu unterstützen..$^{51}$ Indem ein Teil der Opposition einen Kanzler (mit-)wählt, dann aber nicht in die Regierung eintritt, wird ein Minderheitskanzler als Mehrheitskanzler gewählt - »ohne dass es jemand [der Bundespräsident] verhindern könnte.$^{52}$ Schließlich kann auf diesem Wege »das Auflösungsrecht des Bundespräsidenten nach Art. 63 IV Satz 3 bewusst und gewollt ausgeschaltet werden $\ll .{ }^{53}$ So sehr Art. 63 Abs. 4 Satz 3 GG durch seine verfassungsrechtliche Wirkung die »echte« Wahl eines Minderheitskanzlers (den Prozess, bei dem ein Minderheitskanzler als Minderheitskanzler, also mit relativer Mehrheit, gewählt wird) erschwert, so sehr begünstigt er durch seine psychologische Wirkung die »unechte« Wahl eines Minderheitskanzlers (den Prozess, bei dem ein Minderheitskanzler als Mehrheitskanzler, also mit absoluter Mehrheit, gewählt wird): »Gerade von der Gefahr der Bundestagsauflösung durch den Bundespräsidenten geht im konkreten Fall der Druck aus, der einem Bewerber um das Amt des Bundeskanzlers die absolute Mehrheit bei der parlamentarischen Wahl, nicht notwendig aber auch die stetige Unterstützung durch die gleiche Mehrheit verschafft. ${ }^{54}$ Natürlich existiert auch die Möglichkeit, dass ein »echter « Mehrheitskanzler gewählt wird, dieser einige Zeit als solcher amtiert, im Laufe der Wahlperiode jedoch (z.B. durch den Austritt einer Partei aus der Regierungskoalition) die Unterstützung der absoluten Mehrheit verliert und dann als Minderheitskanzler » weiteramtiert ${ }{ }^{55}$ - sofern er nicht durch ein erfolgreiches konstruktives Misstrauensvotum gestürzt wird, zurücktritt oder dem Bundespräsidenten nach einer gescheiterten Vertrauensfrage die Auflösung des Bundestags vorschlägt und dieser dem Ersuchen nachkommt. Dass es bisher

50 Eine ähnliche Regelung wie in Schweden findet sich in Portugal. Dort muss die (bereits ernannte) Regierung bei einer (zwar nicht obligatorischen, aber möglichen und auch üblichen) Abstimmung über das vom Ministerpräsidenten vorgestellte Regierungsprogramm zurücktreten, wenn eine absolute Mehrheit dagegen stimmt (Art. 192 der portugiesischen Verfassung). Vgl. auch Ismayr, »Die politischen Systeme Westeuropas im Vergleich «, aaO. (FN 15), S. 21; Wolfgang Ismayr, »Die politischen Systeme Osteuropas im Vergleich« in: ders. (Hg.), Die politischen Systeme Osteuropas, Wiesbaden 2006, S. 9-69, hier: S. $27 \mathrm{ff}$.

51 Die Mithilfe besteht in diesem Fall also darin, dass Nicht-Regierungsparteien für die Minderheitsregierung stimmen müssen (sie also unterstützen müssen), während sie in den skandinavischen Ländern nur nicht gegen die Minderheitsregierung stimmen dürfen (sie also nur tolerieren müssen).

52 Herzog, »Art. 63 GG«, aaO. (FN 44), Rdnr. 54.

53 Ebd.

54 Ebd.

55 Ebd.

ZfP 56. Jg. 3/2009 
weder den Versuch einer »echten« noch den Versuch einer »unechten« Wahl eines Minderheitskanzlers gegeben hat, zeigt u.a., dass die Bildung einer Minderheitsregierung nicht nur von der verfassungsrechtlichen Struktur, sondern auch von der politischen Kultur eines Landes abhängt, welche in Deutschland in diesem Fall mindestens so ungünstig ist wie die verfassungsrechtlichen Rahmenbedingungen. Schließlich steht man Minderheitsregierungen in Deutschland auf Bundesebene - unter dem Einfluss »der zum Verständnis der politischen Kultur unseres Landes höchst bedeutsamen [Weimarer] Präsidialkabinette der dreißiger Jahre « ${ }^{56}$ - äußerst skeptisch gegenüber.

\section{Stabilität bzw. Abberufbarkeit einer Minderheitsregierung}

$\mathrm{Zu}$ den wohl subtilsten und zugleich eigentümlichsten Konfigurationen des Grundgesetzes zählt, dass nicht nur die Bildung, sondern auch die Abberufung einer Minderheitsregierung erschwert wird. Schließlich kann eine Minderheitsregierung - wie eine Mehrheitsregierung - nur durch ein konstruktives Misstrauensvotum aus dem Amt entfernt werden. Nach Art. 67 Abs. 1 Satz 1 GG kann der Bundestag »dem Bundeskanzler das Misstrauen nur dadurch aussprechen, dass er mit der Mehrheit seiner Mitglieder einen Nachfolger wählt«. Indem ein Minderheitskanzler nur durch die Wahl eines Mehrheitskanzlers abberufen werden kann, ${ }^{57}$ erhält eine Minderheitsregierung einen ganz besonderen verfassungsrechtlichen Bestandsschutz. So könnte z.B. eine rot-grüne Minderheitsregierung (in der 16. Wahlperiode des Deutschen Bundestags) nur abberufen werden, indem CDU/CSU, FDP und Linkspartei gemeinsam einen Bundeskanzler wählen. Das Beispiel macht sehr gut deutlich, dass »[e]ine bestehende Minderheitsregierung, der eine gespaltene Opposition gegenübersteht, [...] mit verfassungsrechtlichen Mitteln nicht ablösbar « ${ }^{58}$ ist. Dies kann aus parlamentstheoretischer Sicht durchaus kritisch beurteilt werden. Nach Karl Loewenstein führt die Regelung des konstruktiven Misstrauensvotums beispielsweise dazu, dass das Parlament entmachtet bzw. dem demokratischen Prozess »die Kehle zugeschnürt ${ }^{59}$ wird. Jedenfalls macht sie deutlich, dass der Parlamentarische Rat - vor dem Hintergrund der » Weimarer Erfahrungen« - bestrebt war, »einen einmal - mit welcher Mehrheit auch immer - gewählten Kanzler für die Legislaturperiode im Amt zu halten ${ }^{60}$

Ein internationaler Vergleich der Verfahren zur Abberufung der Regierung durch das Parlament (aus politischen Gründen) in parlamentarischen Regierungssystemen zeigt,

56 Steffani, »Zukunftsmodell Sachsen-Anhalt? Grundsätzliche Bedenken«, aaO. (FN 3), S. 721.

57 Denkbar ist natürlich auch die Abberufung eines Minderheitskanzlers durch eine »unechte« Wahl eines neuen Minderheitskanzlers (vgl. oben).

58 Renzsch / Schieren, »Große Koalition oder Minderheitsregierung: Sachsen-Anhalt als Zukunftsmodell des parlamentarischen Regierungssystems in den neuen Bundesländern?«, aaO. (FN 3), S. 397.

59 Karl Loewenstein, Verfassungslebre, Tübingen 1959, S. 93.

60 Renzsch / Schieren, »Große Koalition oder Minderheitsregierung: Sachsen-Anhalt als $\mathrm{Zu}-$ kunftsmodell des parlamentarischen Regierungssystems in den neuen Bundesländern?«, aaO. (FN 3), S. 397. 
dass das in Deutschland praktizierte Verfahren eine sehr große Hürde für und damit eine entsprechend starke Absicherung gegen den Sturz einer Minderheitsregierung impliziert. So ist nur in sehr wenigen Ländern ein konstruktives Misstrauensvotum gegen den Regierungschef bzw. die Regierung zur Abberufung der Regierung erforderlich: in Belgien, Polen, Slowenien, Spanien und Ungarn. Weitaus einfacher ist die Abberufung einer Minderheitsregierung wenn, wie in den meisten parlamentarischen Regierungssystemen, zur Abberufung der Regierung nur ein destruktives Misstrauensvotum notwendig ist. ${ }^{61} \mathrm{Am}$ einfachsten ist die Abberufung einer Minderheitsregierung in diesem Fall, wenn für das Misstrauensvotum nicht, wie in Frankreich, Griechenland, Portugal, Schweden oder den meisten osteuropäischen Ländern, eine absolute, sondern, wie in Lettland, Rumänien, ${ }^{62}$ der Ukraine oder den meisten westeuropäischen Ländern (darunter auch Dänemark und Norwegen), ${ }^{63}$ nur eine einfache Mehrheit erforderlich ist. ${ }^{64}$

Die Wahrscheinlichkeit eines (erfolgreichen) Misstrauensantrags hängt natürlich von verschiedenen Faktoren ab, u.a. auch von der in diesem Zusammenhang bestehenden Möglichkeit oder Notwendigkeit einer Parlamentsauflösung bzw. vorzeitigen Neuwahl - v.a. der Möglichkeit der Regierung (evtl. mit Unterstützung des Staatsoberhaupts), vor oder nach einem (erfolgreichen) Misstrauensvotum das Parlament aufzulösen bzw. damit zu drohen. In den skandinavischen Ländern existieren hierzu höchst unterschiedliche Regelungen: Während es in Norwegen generell keine vorzeitige Neuwahl geben kann, hat die Regierung in Dänemark sowie (unter bestimmen Voraussetzungen) auch in Schweden ${ }^{65}$ vor sowie nach einem (erfolgreichen) Misstrauensvotum die Möglichkeit,

61 Schließlich bedarf eine Minderheitsregierung unter der Bedingung des destruktiven Misstrauensvotums einer gewissen Tolerierung im Parlament, während sie unter der Bedingung des konstruktiven Misstrauensvotums nur eine fehlende alternative Regierungsmehrheit benötigt.

62 Dort stimmen beide Kammern in gemeinsamer Sitzung ab.

63 Mit Blick auf Norwegen ist darauf hinzuweisen, »dass es keine eindeutigen Regeln für den Rücktritt einer Regierung gibt. Verfassungsrechtlich verankert ist (mit Wirkung 2009) der zwingende Rücktritt eines Regierungsmitglieds nach einem Misstrauensvotum des Parlaments, was in der Praxis seit 1884 so gehandhabt wurde " (Groß / Rothholz, »Das politische System Norwegens «, aaO. (FN 11), S. 162). Folglich bildete das Misstrauensvotum - wie in Großbritannien und Luxemburg - eine Verfassungskonvention. In manchen Ländern bestehende Konventionen, dass Regierungen bei essenziellen Abstimmungsniederlagen (z.B. beim Haushalt) zurücktreten bzw. Neuwahlen ausschreiben, werden an dieser Stelle prinzipiell nicht berücksichtigt, da diese zum Teil vom Ermessen der Regierungen abhängen und infolgedessen - wie der Fall Dänemark zeigt (Nannestad, »Das politische System Dänemarks «, aaO. (FN 8), S. 77) - Veränderungen unterworfen sind bzw. für die Abberufbarkeit der Regierung in diesem Kontext keinen signifikanten zusätzlichen Erklärungswert bieten. Erwähnung verdienen in diesem Zusammenhang nur die Niederlande. Dort existiert kein »einfaches Misstrauensvotum «, aber die Möglichkeit des Parlaments, die Regierung durch die Ablehnung oder gravierende Änderung eines zentralen Gesetzesvorhabens bzw. des Staatshaushalts faktisch zum Rücktritt zu zwingen (Ismayr, »Die politischen Systeme Westeuropas im Vergleich «, aaO (FN 15), S. 24).

64 Vgl. auch ebd.; Ismayr, »Die politischen Systeme Osteuropas im Vergleich «, aaO (FN 50), S. 30 .

65 Davon bleibt allerdings der Wahlrhythmus unberührt. Schließlich wird der schwedische Reichstag in einem festen Vier-Jahres-Rhythmus gewählt (Jahn, »Das politische System Schwedens«, aaO. (FN 10), S. 114). 
eine Neuwahl auszuschreiben. ${ }^{66}$ In Deutschland besteht für die Bundesregierung weder vor noch nach einem konstruktiven Misstrauensvotum die Möglichkeit, den Bundestag aufzulösen. So ist es ihr z.B. nicht möglich, eine (sich abzeichnende) Abberufung durch die Androhung oder Durchführung von Neuwahlen zu verhindern. Allerdings ist die Hürde zur Abberufung der Regierung durch das konstruktive Misstrauensvotum in Deutschland ohnehin sehr groß.

Der internationale Vergleich macht deutlich, dass die verfassungsrechtlichen Rahmenbedingungen für die Stabilität einer Minderheitsregierung in Deutschland sehr günstig sind, während sie in den skandinavischen Ländern, in denen vermehrt Minderheitsregierungen gebildet werden, verhältnismäßig ungünstig sind. Dies zeigt wiederum, dass die Stabilität einer Minderheitsregierung nicht nur von der verfassungsrechtlichen Struktur, sondern u.a. auch von der politischen Kultur eines Landes abhängt. So waren Minderheitsregierungen - trotz verfassungsrechtlich verhältnismäßig ungünstiger Rahmenbedingungen - in Dänemark, Norwegen sowie Schweden relativ stabil und in Italien, der vierten Republik Frankreichs sowie der Weimarer Republik relativ instabil. ${ }^{67}$ In der Bonner/Berliner Republik waren sie auf Bundesebene wiederum - trotz verfassungsrechtlich sehr günstiger Rahmenbedingungen - nur »krisenhafte Übergangserscheinungen«. Dazu zählen die Minderheitsregierungen von Konrad Adenauer 1962, Ludwig Erhard 1966 und Helmut Schmidt 1982, die nach dem jeweiligen Koalitionsbruch »zurückblieben«, bis kurze Zeit darauf wieder eine (Mehrheits-)Koalition gebildet wurde. Eine »planmäßige « Bildung einer (dauerhaften) Minderheitsregierung gab es in der Bundesrepublik Deutschland bisher nur auf Landesebene. ${ }^{68}$ Allerdings existierten auf Bundesebene nach Wahlen bzw. Regierungskrisen stets mehrheitsfähige Alternativen, was in Zukunft nicht zwingend der Fall sein muss.

\section{Funktionalität bzw. Handlungsfähigkeit einer Minderbeitsregierung}

Während sich die Einsetzbarkeit sowie die Abberufbarkeit (und damit die Stabilität) einer Minderheitsregierung relativ gut beschreiben lassen, ist die Handlungsfähigkeit einer Minderheitsregierung, v.a. die Möglichkeit einer »effizienten Gesetzgebung « ${ }^{69}$ unter den Bedingungen einer Minderheitsregierung, nur schwer einzuschätzen. Schließlich hängt diese stark von der Akteurkonstellation ab - v.a. von der Akteurkonstellation in der Opposition, der bei einer Minderheitsregierung eine völlig neue (entscheidungsrelevante) Bedeutung zuwächst. Da nach Art. 42 Abs. 2 Satz 1 GG für einen Beschluss des Bundestags im Normalfall eine »Mehrheit der abgegebenen Stimmen« - d.h. eine einfache

66 Die Wirkung dieser Option kann nicht pauschal vorhergesagt werden. Sie kann sich prinzipiell - in Abhängigkeit von anderen Faktoren - sowohl stabilisierend als auch destabilisierend auf Minderheitsregierungen auswirken.

67 Renzsch / Schieren, »Große Koalition oder Minderheitsregierung: Sachsen-Anhalt als $\mathrm{Zu}-$ kunftsmodell des parlamentarischen Regierungssystems in den neuen Bundesländern?«, aaO. (FN 3), S. 394.

68 Ebd., S. 394f.

69 Ebd., S. 392. 
Mehrheit - ausreicht, aufgrund der parlamentarischen Praxis bzw. der Gefahr einer alternativen Mehrheit de facto jedoch eine Mehrheit der Mitglieder des Bundestags - d.h. eine absolute Mehrheit - erforderlich ist, muss eine Minderheitsregierung grundsätzlich jenseits der Regierungsfraktion(en) nach einer Mehrheit suchen. Sie ist also in gewisser Weise gezwungen, mit der Opposition zu kooperieren. ${ }^{70}$ Dabei bieten sich grundsätzlich zwei Möglichkeiten:

- erstens das »Quasi-Koalitions-Modell«, bei dem die Minderheitsregierung ein dauerhaftes bzw. formalisiertes Kooperationsverhältnis mit einem Teil der Opposition eingeht. Dabei muss ein Teil der Opposition folglich bereit sein, kontinuierlich (koalitionsähnliche) Kompromisse in Sachfragen mit der Regierung einzugehen, ohne selbst Regierungsvertreter zu entsenden bzw. Regierungsverantwortung zu übernehmen. ${ }^{71}$ Bei diesem Modell weist die Minderheitsregierung Parallelen zu einer (Koalitions-)Mehrheitsregierung auf, weshalb sie sich als »stabile Minderheitsregierung " oder gar »heimliche Mehrheitsregierung « bezeichnen lässt. ${ }^{72}$

- zweitens das »Ad-hoc-Koalitionen-Modell«, bei dem die Minderheitsregierung situative bzw. einzelfallbezogene Kooperationsverhältnisse mit unterschiedlichen Teilen der Opposition eingeht. Dabei wird - die Kooperationsbereitschaft der Oppositionsparteien vorausgesetzt - etwas möglich, was das parlamentarische Regierungssystem im Normalfall verhindert: das Regieren bzw. die Gesetzgebung mit wechselnden Mehrheiten - wobei auch »Oppositionsmehrheiten«, also Mehrheiten gegen die Minderheitsregierung denkbar sind. Bei diesem Modell weist die Minderheitsregierung Parallelen zu einer Regierung in einem präsidentiellen Regierungssystem auf.

Beide Modelle stellen letztlich Idealtypen dar, die in der Realität mit unterschiedlichen Schattierungen und fließenden Übergängen existieren. ${ }^{73}$

70 Die Erfolgsaussichten dieser Kooperation hängen - neben der Anzahl der die Minderheitsregierung tragenden Parteien - u.a. davon ab, ob die Minderheitsregierung nur eine oder mehrere Oppositionsparteien für eine absolute Mehrheit benötigt und ob diese aus dem »eigenen« oder »fremden « Parteienspektrum (»Block «) stammt bzw. stammen (Jahn, »Koalitionen in Dänemark und Norwegen: Minderheitsregierungen als Normalfall«, aaO. (FN 5), S. 230). So wurden z.B. in Schweden, als von der Sozialdemokratischen Arbeiterpartei gebildete Minderheitsregierungen (zwischen 1998 und 2006) nicht mehr (wie früher) nur auf die Unterstützung einer Partei (der Linkspartei), sondern auf die Unterstützung von zwei Parteien (der Linkspartei und der Umweltpartei) angewiesen waren, die Verhandlungen nicht unmaßgeblich komplexer und schwieriger (Jahn, »Das politische System Schwedens «, aaO. (FN 10), S. 114ff.).

71 Eberhard Schütt-Wetschky, »Verhältniswahl und Minderheitsregierungen. Unter besonderer Berücksichtigung Großbritanniens, Dänemarks und der Bundesrepublik Deutschland in: ZParl 18, Nr. 1 (1987), S. 94-109, hier: S. 105f.

72 Christian Starck »Stabile Minderheitsregierung als heimliche Mehrheitsregierung. Zum Magdeburger Modell« in: Jörn Ipsen / Edzard Schmidt-Jortzig (Hg.), Recht - Staat - Gemeinwohl, Köln 2001, S. 157-172, hier: S. 157.

73 Sven Thomas, der eine etwas differenziertere Betrachtung entwickelt hat, unterscheidet am Beispiel des »Magdeburger Modells « zwischen einem Konzept »diskursiver Kooperation «, das auf parlamentarischer Ebene sachpolitische Mehrheiten generiert, einem Konzept »parlamentarischen Verhandlungsmanagements «, das auf parlamentarischer Ebene parteipolitische Mehrheiten generiert, und einem »regierenden Verhandlungssystem «, das auf gouvernementaler Ebene parlamentarische bzw. parteipolitische Mehrheiten generiert (Thomas, »Zur Hand- 
In skandinavischen Ländern sind prinzipiell beide Varianten vorzufinden. In Dänemark gab es zwischen 1945 und 1993 sowohl Minderheitsregierungen mit fester Unterstützung als auch Minderheitsregierungen ohne feste Unterstützung (wobei - gemessen an der Amtszeit - Letztere dominierten), von 1993 bis 2001 nur noch Minderheitsregierungen ohne feste Unterstützung und anschließend wieder beide Varianten (wobei gemessen an der Amtszeit - diesmal Minderheitsregierungen mit fester Unterstützung dominierten). Zur Zeit der bürgerlichen Minderheitsregierungen von 1982 bis 1988 »regierte in einigen Politikbereichen de facto eine alternative Mehrheit im Parlament, da die Regierungen trotz einer größeren Anzahl von Abstimmungsniederlagen in zentralen Bereichen (u.a. der Außen- und Sicherheitspolitik) nicht - wie bis dahin üblich - zurücktraten oder Neuwahlen ausschrieben und die (zur alternativen Mehrheit gehörige) Radikal-liberale Partei nicht bereit war, ein Misstrauensvotum zu unterstützen. ${ }^{74}$ Auch in Schweden existierten sowohl Minderheitsregierungen mit einer »hidden majority « 75 als auch Minderheitsregierungen mit (grundsätzlich) wechselnden Mehrheiten. So konnten Minderheitsregierungen der Sozialdemokratischen Arbeiterpartei in dem bis 1988 bestehenden Zwei-Block-Parteiensystem mit einer »Rückendeckung durch die Kommunistische Partei ${ }^{76}$ rechnen, wenngleich sie bewusst die (aufgrund der »Rückendeckung « nicht zwingend erforderliche) Unterstützung bzw. Kooperation mit Parteien aus dem bürgerlichen Block suchten. Später war dies - aufgrund der abnehmenden Dominanz der Sozialdemokratischen Arbeiterpartei bzw. der damit einer gehenden fehlenden Mehrheit zwischen Sozialdemokratischer Arbeiterpartei und der zur Linkspartei mutierten Kommunistischen Partei sowie der fehlenden Bereitschaft Letzterer zur bedingungslosen Unterstützung Ersterer - nicht mehr möglich. So war die nach der Reichstagswahl 1998 gebildete sozialdemokratische Regierung eine Minderheitsregierung, die auf die Unterstützung der Linkspartei und der Umweltpartei angewiesen war und nur in bestimmten Bereichen (v.a. der Haushaltspolitik) mit deren festen Unterstützung rechnen konnte. Auch in Norwegen konnten sich von der Arbeiterpartei gebildete Minderheitsregierungen - wie zum Teil die Sozialdemokratische Arbeiterpartei in Schweden - auf den »linken Rand « verlassen, während sie mit anderen Parteien themenbezogene parlamentarische Mehrheiten anstrebte und auch erreichte. ${ }^{77}$ Hierbei wird deutlich, dass

lungsfähigkeit von Minderheitsregierungen am Beispiel des >Magdeburger Modells« «, aaO. (FN 4), S. 797ff.). Während sich das erste Konzept dem Ad-hoc-Koalitionen-Modell zurechnen lässt, lassen sich das zweite und dritte Konzept dem Quasi-Koalitions-Modell zuordnen. Bei dem zuletzt genannten Konzept handelt es sich letztlich nur um eine Weiterentwicklung bzw. Extremform der formalisierten Kooperation der Minderheitsregierung mit einem Teil der Opposition, die eine besonders starke Annäherung an das Regieren in einer (Koalitions-)Mehrheitsregierung erkennen lässt.

74 Nannestad, »Das politische System Dänemarks«, aaO. (FN 8), S. 77.

75 Leif Lewin »Majoritarian and Consensus Democracy: the Swedish Experience« in: Scandinavian Political Studies 21, Nr. 3 (1998), S. 195-206, hier: S. 204.

76 Pehle, »Koalitionen in Finnland und Schweden: Fortbestand der Unterschiede trotz Angleichung der Systeme«, aaO. (FN 26), S. 205.

77 Jahn, »Koalitionen in Dänemark und Norwegen: Minderheitsregierungen als Normalfall«, aaO. (FN 5), S. 222. 
beide Modelle in der Realität nicht nur mit unterschiedlichen Schattierungen, sondern auch mit fließenden Übergängen bzw. letztlich in Kombination auftreten können.

Aus demokratietheoretischer Sicht ist das Ad-hoc-Koalitionen-Modell sicherlich weitaus interessanter als das Quasi-Koalitions-Modell. Im Normalfall führt das parlamentarische Regierungssystem - aufgrund dessen Funktionslogik, die das Parlament in eine mit der Regierung verzahnte Parlamentsmehrheit und eine die Opposition bildende Parlamentsminderheit trennt, und des (in der Regel schriftlich in der Koalitionsvereinbarung festgehaltenen) Koalitionsgrundsatzes, ein einheitliches Abstimmungsverhalten zu praktizieren bzw. keine wechselnden Mehrheiten zuzulassen ${ }^{78}$ - zu einem (exekutivdominierten) "geschlossenen « Willensbildungs- und Entscheidungsprozess innerhalb der Regierungsmehrheit: ${ }^{79}$ Beschlüsse des Parlaments sind in diesem Fall in der Regel Entscheidungen der Regierungsmehrheit, die machtpolitisch zusammengehalten wird. Dagegen ermöglicht eine Minderheitsregierung, die über kein dauerhaftes bzw. formalisiertes Kooperationsverhältnis mit einem Teil der Opposition verfügt, einen (parlamentszentrierten) »offenen « Willensbildungs- und Entscheidungsprozess im gesamten Parlament: Beschlüsse des Parlaments sind in diesem Fall Entscheidungen unterschiedlicher Mehrheiten bzw. »Sachkoalitionen «, die (überwiegend) programmatisch zustande kommen. Dadurch ist der legislative Handlungsspielraum nicht mehr - wie bei einer Mehrheitsregierung - durch die Regierungsmehrheit, sondern durch das gesamte Parlament definiert. Die »Entgrenzung « des legislativen Handlungsspielraums kommt einer »Präsidialisierung « des Regierungssystems, d.h. einer Annäherung an die Funktionsweise des präsidentiellen Regierungssystems, ${ }^{80} \mathrm{bzw}$. einer »Parlamentarisierung « der Politik, ${ }^{81}$ d.h. einer (gewissen) Entkoppelung von exekutiver Machtpolitik und parlamentarischer Sachpolitik, gleich ${ }^{82}$ - und impliziert weit reichende demokratietheoretische Konsequenzen. Schließlich führt der Normalfall parlamentarischer Regierungsweise dazu, dass »natürliche Mehrheiten« (Entscheidungen durch - unterschiedliche - Mehrheiten im gesamten Parlament) unterdrückt und »künstliche Mehrheiten « (Entscheidungen durch die Regierungsmehrheit) generiert werden: Existieren bei einer Koalitions-Mehrheitsregierung Differenzen innerhalb der Regierungsparteien ist nur das Festhalten am status quo oder eine Reform in Gestalt eines Kompromisses zwischen den Regierungsparteien, also in Form einer »künstlichen Mehrheit«, möglich. Für »empfindliche Ein-

78 Uwe Kranenpohl, Mächtig oder machtlos? Kleine Fraktionen im Deutschen Bundestag 1949 bis 1994, Opladen 1999, S. 267.

79 Dabei werden politische Entscheidungen bzw. Gesetze(svorhaben) in der Regel von der Regierung initiiert und mit Hilfe der Regierungsmehrheit implementiert - also weitestgehend auf Regierungsebene getroffen.

80 Renzsch / Schieren, »Große Koalition oder Minderheitsregierung: Sachsen-Anhalt als $\mathrm{Zu}-$ kunftsmodell des parlamentarischen Regierungssystems in den neuen Bundesländern?«, aaO. (FN 3), S. 398.

81 Der Begriff der »Parlamentarisierung « wird benutzt, da sich das Parlament in gewisser Weise von der Regierung »emanzipieren « und sich der Schwerpunkt der Gesetzgebungstätigkeit vom Kabinettstisch in das Plenum und die Ausschüsse des Parlaments verlagern würde (ebd., S. 398ff.). Folglich würde die Regierung geschwächt und das Parlament gestärkt werden.

82 Bei einer Mehrheitsregierung sind beide Komponenten miteinander verzahnt, so dass sich mit nahezu jeder Sachfrage im Parlament die Machtfrage für die Regierung stellt. 
bußen an Entscheidungsrationalitä « ${ }^{83}$ sorgt dies auf jeden Fall dann, wenn eine der Regierungsparteien mit einer oder mehreren Oppositionsparteien eine alternative Mehrheit bilden könnte, die weniger auf einem Kompromiss, sondern mehr auf den originären Vorstellungen der Parteien basiert. ${ }^{84} \mathrm{Zu}$ denken sind z.B. an politische Reformen, die in der 16. Wahlperiode des Deutschen Bundestags im Rahmen der »schwarz-roten « Koalition keine Mehrheit gefunden haben, jenseits der Regierungsparteien jedoch eine (z.B. »schwarz-gelb-grüne« oder »rot-gelb-grüne«) Mehrheit gehabt hätten. Die Tatsache, dass der legislative Handlungsspielraum nicht mehr (durch die Regierungsmehrheit) begrenzt wäre, hätte jedoch nicht zwingend zur Folge, dass er sich (durch eine konsequente Nutzung) auch tatsächlich »erweitert«. Schließlich würde die Nutzung des legislativen Handlungsspielraums stark vom Verhalten der Parteien (den Regierungs- und v.a. den Oppositionsparteien) abhängen.

In jedem Fall, d.h. sowohl im Rahmen des Ad-hoc-Koalitionen-Modells als auch im Rahmen des Quasi-Koalitions-Modells, ist fraglich, ob die Oppositionsparteien zu einer Kooperation mit der Minderheitsregierung bereit wären. Dabei ist zu betonen, dass eine gewisse Kooperationsbereitschaft - mit Blick auf essenzielle Entscheidungen (insbesondere mit Blick auf die Feststellung des Haushaltsplans) - bei beiden Varianten unabdingbar ist. Ohne sie würde die Minderheitsregierung nur eine (»beschränkt geschäftsfähige«) geschäftsführende Regierung bilden und die Bundesrepublik letztlich in Richtung Unregierbarkeit abdriften. Fraglich ist natürlich v.a., ob sich die Oppositionsparteien darauf einlassen würden, Ad-hoc-Koalitionen mit der Minderheitsregierung zu bilden. ${ }^{85}$ Dafür sprechen ...

- policy- und (damit verbundene) vote-seeking Motive der Parteien. ${ }^{86}$ So können Parteien das Ziel verfolgen, politische Entscheidungen zu beeinflussen bzw. sachpolitische Forderungen durchzusetzen, ohne dafür die »Kosten« des Regierens tragen zu müssen. Unter »Kosten« des Regierens sind Zustimmungsverluste zu verstehen, »die in Form der im Allgemeinen sinkenden Popularität von Regierungsparteien während ihrer Amtszeit auflaufen $\ll{ }^{87}$ Wichtig ist in diesem Zusammenhang, dass Politikgestaltung auch unabhängig von der Besetzung von Regierungsämtern möglich ist, sich also office- und policy-seeking Motive nicht decken müssen. ${ }^{88}$

83 Gerhard Lehmbruch, zit. in Thomas, "Zur Handlungsfähigkeit von Minderheitsregierungen am Beispiel des >Magdeburger Modells««, aaO. (FN 4), S. 797.

84 Im Idealfall entsteht eine von einer »pivotal party (»einer Partei mit Scharnierfunktion zwischen links und rechts «) geführte Minderheitsregierung, deren Politik einer »auf Inhalte bezogenen Überzeugungsarbeit« und nicht einer »am bloßen Machterhalt ausgerichteten Parteitaktik « folgt (Pehle, »Koalitionen in Finnland und Schweden: Fortbestand der Unterschiede trotz Angleichung der Systeme«, aaO. (FN 26), S. 204).

85 Renzsch / Schieren, »Große Koalition oder Minderheitsregierung: Sachsen-Anhalt als $\mathrm{Zu}-$ kunftsmodell des parlamentarischen Regierungssystems in den neuen Bundesländern?«, aaO. (FN 3), S. 399.

86 Kaare Strøm, Minority Government and Majority Rule, Cambridge 2001.

87 Pehle, »Koalitionen in Finnland und Schweden: Fortbestand der Unterschiede trotz Angleichung der Systeme«, aaO. (FN 26), S. 202.

88 Ebd. 
- »Fliehkräfte in den Parteien« bzw. die Tatsache, dass »gegensätzliche Interessen in einer Partei und gleiche Interessen in verschiedenen Parteien « 89 existieren, die übergreifende sachpolitische Koalitionen (möglicherweise auch quer durch Fraktionen) begünstigen.

Dagegen sprechen ...

- »office-« und (damit verbundene) vote-seeking Motive der Parteien. So können Parteien das Ziel verfolgen, den Wählern die Handlungsunfähigkeit der Minderheitsregierung vor Augen zu führen und sich dadurch als Regierungsalternative zu präsentieren bzw. zu profilieren.

- die »tradierte Parlamentskultur« bzw. die Tatsache, dass jahrzehntelang »eingeübte« Konfliktmuster zwischen Regierungs- und Oppositionsparteien existieren, die übergreifende sachpolitische Koalitionen erschweren.

Die Frage, ob die Oppositionsparteien in Deutschland tatsächlich zu einer Kooperation mit einer Minderheitsregierung bereit wären, v.a. ob sie sich darauf einlassen würden, Ad-hoc-Koalitionen mit einer Minderheitsregierung zu bilden, hängt ganz wesentlich von der politischen Kultur im Allgemeinen bzw. der Parlamentskultur im Speziellen ab.

Ein internationaler Vergleich zeigt, dass sich dieser Faktor in den skandinavischen Ländern, in denen vermehrt Minderheitsregierungen gebildet werden, eindeutig positiv auf die Handlungsfähigkeit von Minderheitsregierungen auswirkt. So ist dort eine breite Akzeptanz, zum Teil sogar eine prinzipielle Bevorzugung von Minderheitsregierungen sowie eine »korporatistische Prägung der Entscheidungsprozesse «, ${ }^{90}$ eine »konsensuelle Regierungspraxis «11 bzw. eine generelle Konsens- und Kompromissbereitschaft seitens der politischen Akteure ${ }^{92}$ festzustellen. Die »konsensuelle Regierungspraxis « in Dänemark, Norwegen und Schweden zeigt sich z.B. darin, dass dort bei politischen Entscheidungen sowohl die Interessengruppen als auch die Oppositionsparteien - über die Ausschüsse der (Arbeits-)Parlamente - generell verhältnismäßig stark eingebunden werden. ${ }^{93}$ In Deutschland dürfte der Faktor politische Kultur bzw. Parlamentskultur auf jeden Fall ungünstiger mit Blick auf die Handlungsfähigkeit einer Minderheitsregierung ausfallen. So sind in Deutschland - im Vergleich zu den skandinavischen Ländern - die Vorbehalte gegenüber einer Minderheitsregierung (historisch bedingt) relativ stark und die politische Konsens- und Kompromissbereitschaft relativ schwach ausgeprägt. Allerdings unterscheidet sich die politische Kultur der Bundesrepublik vehement von der po-

89 Renzsch / Schieren, »Große Koalition oder Minderheitsregierung: Sachsen-Anhalt als $\mathrm{Zu}-$ kunftsmodell des parlamentarischen Regierungssystems in den neuen Bundesländern?«, aaO. (FN 3), S. 400.

90 Pehle, »Koalitionen in Finnland und Schweden: Fortbestand der Unterschiede trotz Angleichung der Systeme«, aaO. (FN 26), S. 203.

91 Jahn, »Koalitionen in Dänemark und Norwegen: Minderheitsregierungen als Normalfall«, aaO. (FN 5), S. 247.

92 Thomas, »Zur Handlungsfähigkeit von Minderheitsregierungen am Beispiel des >Magdeburger Modells««, aaO. (FN 4), S. 796.

93 Jahn, »Koalitionen in Dänemark und Norwegen: Minderheitsregierungen als Normalfall«, aaO. (FN 5), S. 220, 245; Pehle, »Koalitionen in Finnland und Schweden: Fortbestand der Unterschiede trotz Angleichung der Systeme«, aaO. (FN 26), S. 203. 
litischen Kultur der Weimarer Republik. Sie ist zwar von den »Weimarer Erfahrungen« geprägt, weißt aber im Gegensatz zur Weimarer Republik eine äußerst stabile demokratische Kultur auf - die im Fall einer Minderheitsregierung zwar kein Regierbarkeitsproblem, aber wohl doch ein Demokratieproblem verhindern würde.

Die Funktionalität einer Minderheitsregierung würde in Deutschland auf Bundesebene allerdings auch durch die Existenz einer (starken) zweiten Kammer eingeschränkt werden. Schließlich ist die Steuerungsfähigkeit bei Zweikammersystemen (bei denen die zweite Kammer in der Gesetzgebung in allen oder vielen Bereichen gleichberechtigt neben der ersten Kammer steht) grundsätzlich niedriger als bei (faktischen) Einkammersystemen..${ }^{94}$ Somit hängt die Handlungsfähigkeit einer Minderheitsregierung auch vom Kammersystem ab.

Ein internationaler Vergleich zeigt, dass sich das Kammersystem in den skandinavischen Ländern, in denen vermehrt Minderheitsregierungen gebildet werden, eindeutig positiv auf die Handlungsfähigkeit von Minderheitsregierungen auswirkt. So existieren in Dänemark, Norwegen und Schweden (faktische) Einkammerparlamente. In Deutschland würde die Handlungsfähigkeit einer Minderheitsregierung durch die Existenz des Bundesrats mit großer Wahrscheinlichkeit nicht unerheblich eingeschränkt werden. Zum einen ist der Anteil zustimmungsbedürftiger Gesetze - auch nach der Föderalismusreform von 2006 - relativ groß und die Wahrscheinlichkeit, dass eine Minderheitsregierung den Bundesrat »kontrolliert«, verhältnismäßig klein. Zum anderen kann bei einer Minderheitsregierung unter Umständen auch das Veto des Bundesrats gegen Einspruchsgesetze vom Bundestag nicht zurückgewiesen werden. Schließlich bedarf die Zurückweisung eines Einspruchs, ...

wenn dieser mit der »Mehrheit der Stimmen des Bundesrats « beschlossen wurde, einer »Mehrheit der Mitglieder des Bundestags«, also einer absoluten Mehrheit (Art. 77 Abs. 4 Satz 1 GG), während der Gesetzesbeschluss nur eine »Mehrheit der abgegebenen Stimmen«, also eine einfache Mehrheit, erfordert (Art. 42 Abs. 2 Satz 1 GG). ${ }^{95}$

wenn dieser mit der »Mehrheit von mindestens zwei Dritteln« der Stimmen des Bundesrats beschlossen wurde (was gerade bei einer Minderheitsregierung durchaus möglich ist), einer »Mehrheit von zwei Dritteln, mindestens der Mehrheit der Mitglieder des Bundestags « (Art. 77 Abs. 4 Satz 2 GG).

Infolgedessen kann der Bundesrat bei einer Minderheitsregierung ein noch mächtigerer und bedeutenderer Vetospieler sein als bei Mehrheitsregierungen. An dieser Stelle muss allerdings darauf hingewiesen werden, dass auch viele Mehrheitsregierungen in der

94 Jahn, »Koalitionen in Dänemark und Norwegen: Minderheitsregierungen als Normalfall«, aaO. (FN 5), S. 220.

$95 \mathrm{Da}$ es bei einer Minderheitsregierung - im Gegensatz zu einer Mehrheitsregierung - nicht unwahrscheinlich ist, dass sie bei Gesetzen zwar eine Mehrheit der abgegebenen Stimmen, aber keine Mehrheit der Mitglieder des Bundestags erreicht, kann die Notwendigkeit einer absoluten Mehrheit zur Zurückweisung eines Einspruchs des Bundesrats in diesem Fall eine (nicht unmaßgebliche) machtpolitische Bedeutung entfalten. Während sie also bei einer Mehrheitsregierung in der Verfassungspraxis letztlich keine Relevanz hat, kann sie bei einer Minderheitsregierung zu einer faktischen »Hürde« im Gesetzgebungsprozess avancieren. 
Geschichte der Bundesrepublik keine Mehrheit im Bundesrat hatten. Selbst die zweite Große Koalition hat nach der Bildung der schwarz-gelben Landesregierung in Hessen 2009 keine Mehrheit mehr im Bundesrat.

Der internationale Vergleich macht deutlich, dass die Rahmenbedingungen für die Handlungsfähigkeit einer Minderheitsregierung, die politische Kultur bzw. Parlamentskultur sowie das Kammersystem, in Deutschland auf Bundesebene verhältnismäßig ungünstig sind, während sie in den skandinavischen Ländern, in denen vermehrt Minderheitsregierungen gebildet werden, sehr günstig sind.

Wie die Kooperationsbereitschaft der Oppositionspartien bzw. des Bundesrats bei einer Minderheitsregierung in Deutschland konkret ausfallen würde, ist generell nur schwer prognostizierbar. Fest steht nur, dass weder »eine kompromissbereite Partnerschaft beim Verabschieden von für das Land bedeutsamen Gesetzesvorhaben ${ }^{96}$ noch »eine vollständige Blockade der Gesetzgebung « $97 \mathrm{zu}$ erwarten wäre. Schließlich gibt es Gesetze, z.B. Gesetze, die Steuererhöhungen zur Folge haben oder aus anderen Gründen unpopulär sind, denen eine Opposition kraft Natur der Sache ablehnend gegenüberstehen muss, aber auch Gesetze, z.B. Gesetze, die Steuersenkungen zur Folge haben oder aus anderen Gründen populär sind, »denen sich erfahrungsgemäß keine Opposition entziehen kann «. ${ }^{98}$ Grundsätzlich ist festzuhalten, dass die Handlungsfähigkeit einer Minderheitsregierung in Deutschland im Vergleich zu einer Mehrheitsregierung nicht unmaßgeblich beschränkt, aber nicht unbedingt völlig eingeschränkt wäre. In diesem $\mathrm{Zu-}$ sammenhang ist auch darauf hinzuweisen, dass eine Minderheitsregierung verschiedene Möglichkeiten hätte, ihren grundsätzlich eingeengten Handlungsspielraum kreativ zu gestalten bzw. subtil auszudehnen. So könnte sie versuchen, »die Zahl der für ihre Politik unumgänglichen Gesetze so weit wie möglich zu reduzieren « ${ }^{99}$ und z.B. durch die »umfassende Ausnutzung sämtlicher schon vorhandenen Verordnungsermächtigungen «100 die Regierungspolitik so zu gestalten, dass sie mit möglichst wenigen (förmlichen) Gesetzen auskommt. ${ }^{101}$

96 Steffani, »Wer trägt die Verantwortung? Wider die wissenschaftliche und politische Verharmlosung des >Magdeburger Modells««, aaO. (FN 3), S. 189.

97 Herzog, »Art. 63 GG«, aaO. (FN 44), Rdnr. 59.

98 Ebd.

99 Ebd., Rdnr. 58.

100 Ebd., Rdnr. 57.

101 Eine kaum gangbare bzw. wenig Erfolg versprechende Option stellt indessen der Weg über den Gesetzgebungsnotstand dar (ebd., Rdnr. 56) - also über das außerordentliche Gesetzgebungsverfahren, das vom Bundespräsidenten nach einer gescheiterten Vertrauensfrage des Bundeskanzlers unter bestimmten Voraussetzungen eingeleitet werden kann und bei dem ein Gesetz »allein durch Zusammenwirken von Bundesregierung, Bundesrat und Bundespräsi- 


\section{Fazit}

Es konnte gezeigt werden, dass die Rahmenbedingungen für die Bildung einer Minderheitsregierung in den skandinavischen Ländern, in denen vermehrt Minderheitsregierungen gebildet werden, (erwartungsgemäß) günstiger sind als in Deutschland. Dennoch gäbe es in Deutschland verschiedene Möglichkeiten, eine Minderheitsregierung einzusetzen. Außerdem wären die Rahmenbedingungen für die Stabilität einer Minderheitsregierung in Deutschland (wider erwarten) günstiger als in den skandinavischen Ländern. Die Rahmenbedingungen für die Handlungsfähigkeit einer Minderheitsregierung wären in Deutschland indessen ungünstiger als in den skandinavischen Ländern. Allerdings ist festzuhalten, dass die Handlungsfähigkeit einer Minderheitsregierung in Deutschland im Vergleich zu einer Mehrheitsregierung zwar nicht unmaßgeblich beschränkt, aber nicht unbedingt völlig eingeschränkt wäre. Schließlich würde die Bildung einer Minderheitsregierung in der Bundesrepublik Deutschland auf Bundesebene aufgrund der strukturellen und kulturellen Unterschiede zur Weimarer Republik ${ }^{102}$ zu einer sehr stabilen und nicht unbedingt zu einer ineffizienten, zumindest nicht zu einer völlig ineffizienten, die Demokratie destabilisierenden Regierung führen.

\section{Zusammenfassung}

Die Ressentiments gegenüber einer Minderheitsregierung auf Bundesebene sind in Deutschland äußerst stark. In einem Fünf-Parteiensystem mit einer fehlenden Mehrheit für eine Kleine Koalition aus zwei Parteien erscheint jedoch eine grundsätzliche Diskussion über eine Minderheitsregierung als Regierungsalternative sinnvoll, da eine Dreierkoalition eine unwahrscheinliche bzw. unbeständige sowie eine Große Koalition eine nicht unumstrittene bzw. unproblematische Konstellation darstellt. Der Beitrag untersucht die Kreation bzw. Einsetzbarkeit, Stabilität bzw. Abberufbarkeit und Funktionalität bzw. Handlungsfähigkeit einer Minderheitsregierung in Deutschland auf Bundesebene im internationalen Vergleich. Dabei wird jeweils geklärt, ob die - strukturellen und kulturellen - Rahmenbedingungen für Minderheitsregierungen in Deutschland (erwartungsgemäß) ungünstiger sind als in Dänemark, Norwegen und Schweden, wo vermehrt Minderheitsregierungen gebildet werden.

\section{Summary}

The objections against minority governments in Germany on the federal level are very strong. However, in a five-party-system with a missing majority for a minimal winning coalition made up of a big and a small party, the discussion about a minority government is necessary, as three-party coalitions are very unlikely as well as unstable and grand

dent zustande [kommt] (Klaus Stern, Das Staatsrecht der Bundesrepublik Deutschland, Band II, München 1980, S. 258).

102 Renzsch / Schieren, »Große Koalition ohne Alternative?«, aaO. (FN 3), S. 188. 
coalitions are problematic in many respects. The paper investigates the creation, stability and functionality of minority governments in Germany on the federal level in comparison to Denmark, Norway and Sweden, where minority governments are regularly built, and gives an answer to the following question: Is the structural and cultural framework for minority governments in Denmark, Norway and Sweden - as expected - really better than in Germany?

Gerd Strohmeier, Minority Governments in Germany on the Federal Level - Crisis or Chance? Findings from a Comparative Perspective

ZfP 56. Jg. 3/2009 